\title{
Teste de esforço: alterações do segmento ST restritas à fase de recuperação
}

\author{
J . A. Oliveira Fo, B. luna Fo, T. Salles, R. Brito, L. Palma, A. Barbieri, J . L. V. Herrmann, e. Martinez Fo
}

Disciplina da Cardiologia - Setor de Ergometria e Reabilitação da Escola Paulista de Medicina, São Paulo, SP.

\begin{abstract}
Resumo - Овj etivo. Determinar a incidência de doença coronária aterosclerótica (DCA) e/ou isquemia miocárdica em pacientes (pt) com alterações do segmento ST restritas à fase de recuperação (ASTRR) do teste ergométrico.

Casuística e Método. Estudo retrospectivo em 19 pacientes não consecutivos com ASTRR, através da cinecoronariografia e/ou cintigrafia de esforço planar. Amostra de 18 homens, uma mulher, com idade de $58 \pm 9$ anos, 18 sintomáticos.

Resultados. ASTRR correspondem a segmentos ST infra-desnivelados de 1 a $4 \mathrm{~mm}$ da linha de base, com inversão da onda $T$ durante a recuperação precoce (2pt), tardia (14 pt). Foi documentada DCA em 14 pt (nove submetidos à cirurgia de revascularização); miocardiopatia hipertensiva
\end{abstract}

\section{INTRODUÇÃO}

Nos primórdios do teste ergométrico os registros el etrocardiográficos eram realizados apenas após o exercício devido à dificuldades técnicas ${ }^{1,2}$. Com o desenvolvimento da monitorização contínua, os traçados durante o exercício passaram a ser valorizados e a maioria dos autores da atualidade tem destacado as alterações do segmento ST durante esforço como preditivas da doença coronária ${ }^{3,4,5}$. Estudos mais recentes têm demonstrado o val or das alterações de ST restritas à fase de recuperação (ASTRR) no diagnóstico da doença coronária em pacientes sintomáticos $\cos ^{6-10} \mathrm{e}$ assintomáti $\cos ^{11}$. A fisi opatologia destas alterações permanece obscura; tem sido relacionada a espasmo coronário $0^{12}$ à níveis el evados de catecolaminas plasmáticas ${ }^{6,13} \mathrm{e}$ à hipopotassemia ${ }^{14,15,16}$. Descreveram-se abolições destas respostas após angioplastia coronária e correção da hipopotassemia ${ }^{15}$.

$\mathrm{Na}$ prática clínica diária, ASTRR tem sido encontrada com certa freqüência, registrando-se na literatura especializada prevalência entre 2,3 a 7,9\% $\%^{6-8}$. Entretanto, dado ao pequeno número de publicações ${ }^{6-9}$, ASTRR não tem sido devidamente valorizada, permanecendo a investigação cardiológica restrita apenas aos casos de maior gravidade.

Neste estudo retrospectivo, descrevemos uma com coronárias normais em 3 pt e prolapso de válvula mitral em 1 pt. Em 13 casos a cinecoronariografia e/ou cintilografia de esforço foram realizados dentro de seis meses do Teste Ergométrico, sendo a presença de DCA e ou isquemia miocárdica detectadas em 8 pt. Em 3 pt, testes ergométricos sucessivos mostraram ASTRR reprodutíveis em dois casos.

Conclusão. Os autores relatam a elevada prevalência de DCA e ou hipoperfusão transitória na cintigrafia de esforço em homens sintomáticos de meia idade com ASTRR no teste de esforço.

Unitermos: Teste de esforço. Cintigrafia de esforço. Doença coronária aterosclerótica.

amostra de pacientes não-sucessivos, portadores de alterações de ST restritas à fase de recuperação. É feita revisão da literatura, a qual mostra escasso número de publicações ${ }^{6-9}$ e citações $^{10-12}$ relativas à ASTRR.

\section{MÉTODO E CASUÍSTICA}

Em levantamento retrospectivo, entre 1986 e 1993, detectaram-se 32 casos não-consecutivos de pacientes em cujo TE existiam alterações de ST restritas à fase de recuperação (ASTRR). Em 13 casos, ASTRR não foram valorizadas pelos médicos assistentes e os respectivos pacientes não foram investigados quanto à presença ou não de cardiopatia. Este estudo se baseia nos 19 casos submeti dos à investigação clínica (tabela 1). N ossa casuística compreendeu 18 homens e uma mulher com idade de $58 \pm 9$ anos; 18 pacientes eram sintomáticos, com sintomas de dor torácica. E m 18 pacientes havia estudo cinecoronariográfico; em oi to casos o intervalo de tempo entre a ci necoronariografia e o TE era inferior a seis meses (casos $1,2,3,4,5,6,7,8)$. Doze pacientes tinham se submetido à cintigrafia de esforço; em seis casos (casos $8,9,10,11,12,13)$ a cintigrafia foi realizada em período inferior a seis meses do TE.

Os TE foram limitados por sintomas, em proto- 


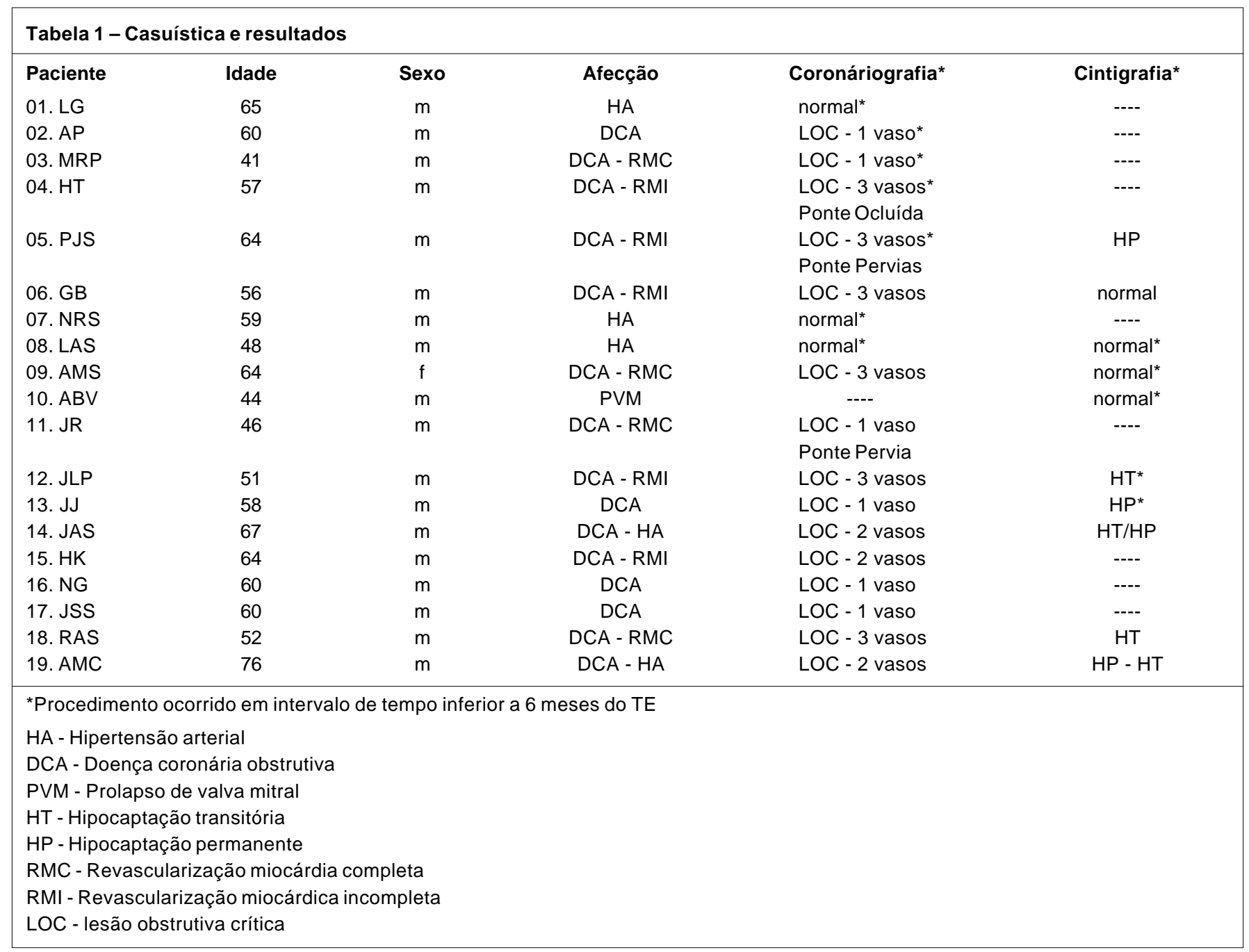

colo de Bruce (17 casos) ou Ellestad (2 casos). Os registros el etrocardi ográficos foram realizados em CM5, D2 modificado e V2. Consideraram-se significativos os desníveis de segmento ST iguais ou superiores a $1,0 \mathrm{~mm}$, a despeito de morfologia ascendente, descendente ou horizontal. Os testes foram realizados em esteira FUNBEC, com registros em cardi oscópi o e el etrocardiógrafo FU NBEC. Dez pacientes realizaram TE após a interrupção dos medicamentos, por período de tempo suficiente para cessação do efeito. Outros pacientes realizaram TE em vigência de medicação: nitratos (cinco casos), antagonistas de cálcio (três casos), betabloqueadores (três casos) e inibidores da ECA (um caso). Um caso não fazia uso de medicação, tendo realizado o TE para avaliação da capacidade física. As cintigrafias de esforço foram planares com imagens em posteroanterior, oblíqua anterior esquerda (45) el ateral esquerda, com 400.000 contagens por imagem, utilizando-se GAMMATOME 9.000 CGR. No caso 13 utilizou-se 6-metoxi-isobutil-isonitrila (MIBI) marcado com tecnécio (99mTc) com injeções de 296 MBq ( $8 \mathrm{mCi}$ ) em repouso e de 888 MBq (24 $\mathrm{mCi}$ ) no pico do esforço com intervalo de quatro horas entre as mesmas; as respectivas aquisições foram realizadas duas horas após as injeções. Nos demais casos utilizou-se táli o ( ${ }^{201} \mathrm{TI}$ ) com injeção de $111 \mathrm{M} \mathrm{bq}$ (3 mCi) no pico do esforço e aquisi ções 8 minutos e quatro horas após o pico do esforço.

As cinecoronáriografias foram realizadas pela técnica de Sones, utilizando-se projeções em oblíqua anterior direita, oblíqua anterior esquerda e perfil esquerdo. Consideraram-se críticas as obstruções iguais ou superiores a $70 \%$ do diâmetro vascular.

\section{RESULTADOS}

Os resul tados estão relacionados na tabel a 1. As alterações do segmento ST ocorreram na fase de recuperação tardia (após o 4으 minuto de recuperação) em 14 casos; na fase de recuperação precoce (entre o $1^{\circ}$ e o $4^{\circ}$ mi nuto da recuperação) em 1 caso e permaneceram durante toda a recuperação em 


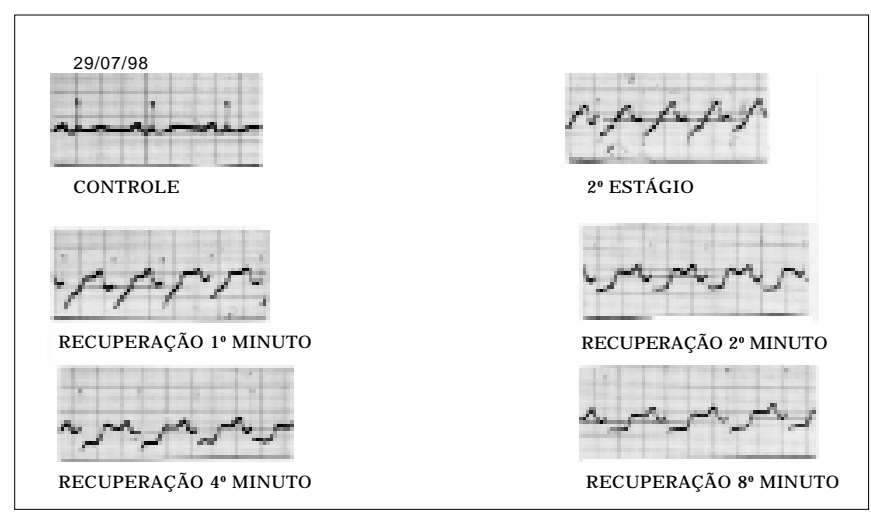

Fig. 1 - TE caso 2

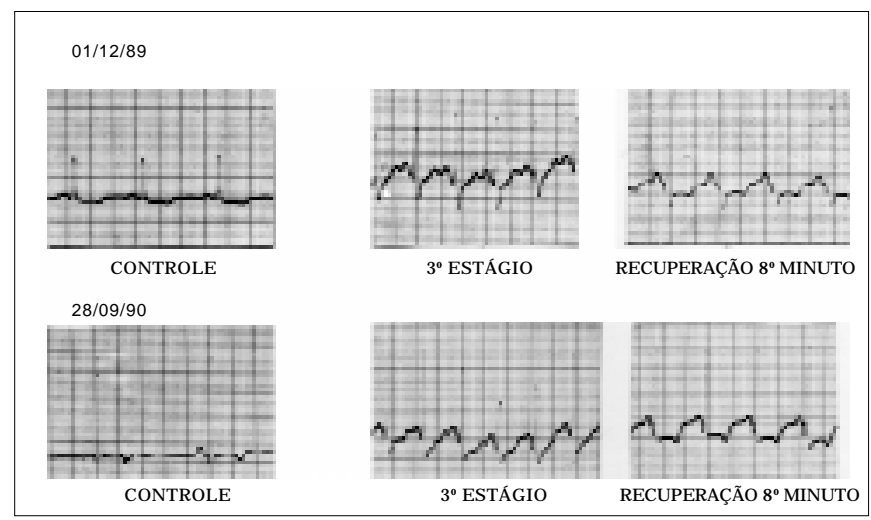

Fig.2 - TE caso 8

quatro casos. O segmento ST apresentou desnivelamento de 1.0 a $1.9 \mathrm{~mm}$ (sete casos); de 2.0 a $2.9 \mathrm{~mm}$ (dez casos); de 3.0 a $3.9 \mathrm{~mm}$ (um caso) e de 4.0 a $4.9 \mathrm{~mm}$ (um caso). A morfologia das ASTRR mostrou segmento ST retificado (sete casos), descendente (três casos), arredondado tipo de ação digitálica (três casos), de convexidade superior (quatro casos), ascendente (um caso) e supradesnivelado (um caso). Três pacientes com ST de convexidade superior apresentavam doença coronária aterosclerótica (DCA) comprovada por cinecoronariografia. Dezoito pacientes eram classe funcional I (NYHA); um caso classe funcional II (NYHA). A capacidade aeróbica variou de 12.5 a $38.8 \mathrm{ml} \mathrm{O}_{2} /$ $\mathrm{Kg} / \mathrm{min}$., sendo a média $25.1 \mathrm{ml} \mathrm{O}_{2} / \mathrm{Kg} / \mathrm{min}$. Em oito casos (casos $1,2,3,4,5,6,7,8$ ) a angiografia coronária tinha se realizado em intervalo inferior a seis meses do TE, correspondendo a três pacientes com hipertensão arterial e coronárias normais, a um paciente com DCA uni-arterial e a quatro pacientes revascularizados (revascularização completa com pontes pérvias, um caso; revascularização incompleta com pontes pérvias, dois casos; revascularização incompleta com ponte ocluída, um caso). E m seis casos $(8,9,10,11,12,13)$ a cintigrafia de esforço tinha se realizado em intervalo inferior a 6 meses do TE com resultados

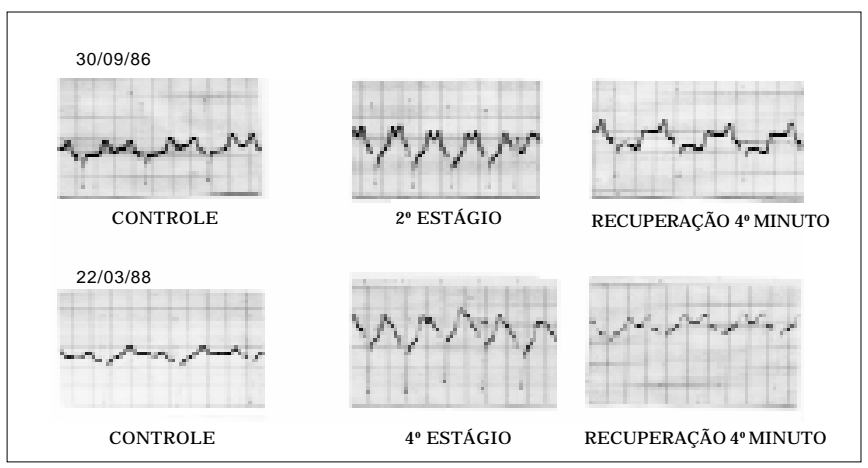

Fig. 3 - TE caso 11

normais em três casos, hipocaptação transitória em dois casos e hipocaptação persistente em um caso. $\mathrm{E} \mathrm{m} 13$ casos nos quais a coronariografia e/ou a cintigrafia realizaram-se em intervalo inferior a 6 meses do teste ergométrico, comprovaram-se lesões obstrutivas e/ou deficiência de perfusão em oito casos. Nos prontuários médicos haviam TE seqüenciais em três casos. Pacientes 3 e 8 apresentaram, respectivamente, três e dois TE em seqüência com ASTRR. Paciente 11 apresentou ASTRR em um exame, que desapareceu noTE seguinte. Exemplos representativos estão nas figuras 1 (caso 2 ), 2 (caso 8) e 3 (caso 11).

\section{DISCUSSÃO}

$\mathrm{Na}$ literatura especializada a ocorrência de ASTRR após o TE é reduzida: $2.3 \%(\mathrm{~K}$ arnegis et al. $\left.{ }^{7}\right), 3.1 \%$ (Savage et al. $\left.{ }^{6}\right), 7.9 \%($ Lachterman et al. $\left.{ }^{8}\right)$. Em nosso material, em uma busca não sistemática em cerca de 8000 TE, detectamos 30 casos. Em 58 aviadores assintomáticos, com TE isquêmico e submeti dos à coronariografia, $F$ roelicher et al. descreveram 15 pacientes portadores de AIRR, dos quais seis apresentavam DCA grave comprovada por cinecoronariografia. No estudo POSCH, Karnegis et al. ${ }^{7}$ analisaram 328 pacientes com infarto prévio e TE isquêmico; em 19 casos relataram AIRR. Não se detectaram indicadores capazes de discriminar estes casos com relação a idade, sexo, el etrocardiograma de repouso, arteriografia coronária, ventriculografia, comportamento de pressão arterial e freqüência cardíaca durante TE e tempo de exercício. Savage et al. ${ }^{6}$ descreveram 62 pacientes com ASTRR em 2.000 cintigrafias de esforço associadas ao TE convencional nos quais ocorreram anomalias de perfusão em $60 \%$ dos casos. A coronariografia foi realizada em 26 casos constatando-se DCA grave em 23 pacientes. Neste estudo, a população não foi clinicamente caracterizada e os critérios de indicação da coronariografia 
não descritos; é possível que os casos de maior risco tenham sido encaminhados à estudo hemodinâmico. Lachterman et al . ${ }^{8}$ analisaram 168 pacientes com TE isquêmico em população de 328 homens sintomáticos de meia idade que tinham sido submetidos à coronariografia. ASTRR foram relatados em 26 casos (valor preditivo positivo de $84 \%$, sensibilidade $8 \%$ e especificidade de $95 \%$, para DAC grave e valor preditivo positivo $19 \%$, sensibilidade de $8 \%$ e especificidade de $92 \%$ para lesões triarteriais e ou lesões de TCE). Tsuchihashi et al. ${ }^{9}$ estudaram 43 pacientes com ASTRR; em 18 casos a cintigrafia de esfor ço era isquêmica, com angina de peito espontânea (78\%), angina instável (33\%), infarto prévio (27\%) e lesões críticas de ADA (94\%). Em 25 casos, sem sinais de isquemia na cintigrafia de esforço, ocorreram $12 \%$ de angina espontânea, estando os eventos coronários praticamente ausentes. A angioplastia coronária aboliu $64 \%$ da ASTRR. Ellestad ${ }^{10}$ relatou a evolução de 308 indivíduos com alterações isquêmicas 3 a 8 minutos após a interrupção do exercício duranteTE, verificando que esta resposta era um indicador fraco, porém definido de futuros eventos coronários.

A fisiopatologia das alterações do segmento ST restritas à fase de recuperação do TE permanece obscura não se conhecendo os aspectos hemodi nâmicos, metaból icos e el etr ofi siológi cos deste evento ${ }^{10}$. Tem se atribuído à queda do débito cardíaco secundária a diminuição do retorno venoso e do fluxo coronário ${ }^{14}$. Deamsdale et al. ${ }^{13}$ observaram el evações séricas de adrenalina em três vezes e de noradrenalina em 10 vezes os valores de repouso em jovens sadios durante os três primeiros minutos após O TE; estes aumentos foram também detectados, em menor intensidade, até o sexto minuto de recuperação, quando as medi das foram interrompidas, Maseri et al. ${ }^{12}$ relataram em pacientes com angina vasoespástica depressão do segmento ST durante ou imediatamente após oTE em 14 dos casos. Georgopoulos et al. ${ }^{15}$ mostraram que as alterações el etrocardiográficas de hipocalemia podiam aparecer apenas no el etrocardiograma registrado após o exercício. Exercitaram-se nove pacientes hipertensos, após depleção de potássio através de diuréticos; durante a fase de recuperação os traçados mostraram sinais evidentes de hipopotassemia, sendo em oito casos compatíveis com testes anormais por desnível dosegmentoST. Estas alterações reverteram com reposi ção de potássio. E m nosso relato, em portadores sintomáticos de ASTRR, ocorreu el evada prevalência de lesões coronárias críticas e ou hipocaptação transitória na cintigrafia de esforço. Recentemente, as ASTRR foram consideradas respostas anormais do segmento ST no TE pelo Consenso Nacional de Ergometria ${ }^{16}$.
Com relação à reprodutibilidade das ASTRR, não existem dados na literatura. É possível que as ASTRR apresentem reprodutibilidade satisfatória levando-se em conta nossos resultados.

\section{CONCLUSÕES}

O real valor das ASTRR no diagnóstico da DCA permanece ainda não definido. Na prática clínica diária tais achados muitas vezes não têm sido valorizados e devidamente investigados, como ocorreu em nosso levantamento. Os resultados do presente estudo e outras publi cações têm mostrado que estas alterações poderiam representar um indicador confiável da DCA. Entretanto, o número de casos devidamente avaliados é pequeno, talvez pela descrença dos clíni cos ao se depararem com os mesmos. Estudos multicêntricos discriminando estes achados em população sintomática e assintomática de baixa, média e alta prevalência de doença coronária, serão necessários para esclarecer o significado clínico e a etiopatogenia das alterações do segmento ST restritas à fase de recuperação do TE.

\section{SUMMARY}

\section{Exercise testing: abnormal ST segments res- trict to recovery phase}

Purpose. To determine the incidence of atherosclerotic coronary artery di sease (CAD) and or myocardial ischemia in patients (pt) with abnormal ST segments restrict to recovery phase (RRAST) of exercise testing (ET).

Material and Method. Retrospectivestudy in 19 non consecutive pt with RRAST, related to coronary arteriography or exercise planar scyntillography (18 men, $58 \pm 9$ years, 18 asymptomatic).

RESULTS. RRAST corresponded to ST segment depression from 1 to $4 \mathrm{~mm}$, with $\mathrm{T}$ inversion during early recovery (2pt); late (14 pt) or both (4pt). It was documented CAD (14 pt and 9) with artery-by-pass surgery); hypertensive myocardiopathy with normal coronary ( $3 \mathrm{pt})$, and mitral prolapse valve (1 pt). In 13 pt with coronary arteriography or exercise scyntillography, within the first 6 months from exercise testing, myocardial ischemia was confirmed in 8 pt in 3 pt, successive exercise testing showed RRAST reproductive in 2 cases.

CONCLUSION. The authors report the high incidence of CAD and or transitory hypoperfusion during myocardial scyntillography in symptomatic men with middleage with RRAST during exercisetesting. [Rev Ass Med Brasil 1999; 45(2): 137-41.]

KEY WORDS: Exercise testing. Exercise scyntillography. Coronary artery disease. 


\section{REFERÊNCIAS BIBLIOGRÁFICAS}

1. Feil H, Siegel M - Eletrocardiographic changes during attacks of angina pectoris. Amer J Med 1928; 175: 255-60.

2. Riseman J EF, Waller J V, Brown MG - The el etrocardiogram during attacks of angina pectoris; its characteristics and diagnostic significance. Amer Heart J 1940; 19: 683-707.

3. Oliveira F.o J A, Pfeferman A, Bocanegra J A, Santos F.o DV, Barcellini A - O teste ergométrico. Arq bras Cardiol 1983; 40:341-51.

4. Detrano R, Froelicher VF - Exercise testing. U ses and limitations. Progr cardiov Dis 1988; 23: 173- 201.

5. Fletcher GF, Balady G, Froelicher VF, et al. - Special report: Exercise standards. Circulation 1995; 91: 580-615.

6. Savage MP, Squares LS, Dopkins J T, Raichlen J S, Park CH, Chung EK - U sefulness of ST - segment depression as a sign of coronary artery disease confined to the exercise recovery period. Am J Cardiol 1987; $60: 1.405$ - 6.

7. Karnegis J M, Matts J , Tuna N, Amplatz K - Comparison of exercise - positive with recovery - positive treadmill graded exercise test. Am J Cardiol 1987; $60: 544$-7.

8. Lachterman B, Lehman KG, Abrahamson D, Froelicher VF "Recorvery Only" ST - segment depression and the preditive accuracy of the exercise test. Ann int Med 1990; 112: 11-6.

9. Tsuchihashi K, Hikita N, Satoh N, NagaoK, Tanaka S, Timura $\mathrm{O}$ - I solated post exercise delayed ST depression as a sign of severe ischemia. Can J Cardiol 1992; 8: 1.050-54.

10. Ellestad MH - Pruebas de Esfuerzo. Barcelona: Ediciones Consulta, 1988: 238 - 41.

11. Froelicher VF, Y anourtz FB, Thompson AJ , Lancaster MC The correlation of coronary angiography and the el ectrocardiographicresponsetomaximal treadmill testing in 76asymptomatic men. Circulation 1973; 48: 597 - 604.

12. Maseri A, Severi S, Nes ML, Abbate A, Chierchia S, Marzilli M, Ballestra AM, Parodi O, Biagini A, DisatanteA - "Variant" angina: one aspect of a continuous spectrum of vasospastic myocardial ischemia. AmJ Cardiol 1978; 42: 1.019-35.

13. DimsdaleJ E, Hartleiy H, Guineiy T, Ruskin J M, Greenblatt D - Post exercise peril. J AMA 1984; 251:630-2.

14. Detry J R - Exercise testing and training in coronary heart disease. Baltimore: Willians \& Wilkins, 1973:55.

15. Georgopoulus AJ , Proudifit WL, Pagel H - Effect of exerciseon electrocardiograms of patients with low serum potassium. Circulation 1961; 23: 567-72.

16. Sociedade Brasileira de Cardiologia. Consenso Nacional de Ergometria. Arq bras Cardiol 1995; 189-211. 